\title{
Cost/efficacy evaluation of the technologies applied to video- assisted thoracoscopic surgery lobectomy
}

\author{
Cecilia Menna ${ }^{1}$, Mohsen Ibrahim ${ }^{1}$, Erino Angelo Rendina ${ }^{1}$, Federico Venuta $^{2}$, Claudio Andreetti $^{1}$ \\ ${ }^{1}$ Division of Thoracic Surgery, Sant'Andrea Hospital, Faculty of Medicine and Psychology, ${ }^{2}$ Division of Thoracic Surgery, Policlinico Umberto I \\ Hospital, Faculty of Pharmacy and Medicine, University of Rome "Sapienza", Rome, Italy \\ Contributions: (I) Conception and design: C Andreetti, C Menna, M Ibrahim, EA Rendina; (II) Administrative support: C Menna; (III) Provision of \\ study materials or patients: C Menna, F Venuta; (IV) Collection and assembly of data: C Menna; (V) Data analysis and interpretation: C Menna; (VI) \\ Manuscript writing: All authors; (VII) Final approval of manuscript: All authors. \\ Correspondence to: Claudio Andreetti. Division of Thoracic Surgery, Sant'Andrea Hospital, Faculty of Medicine and Psychology, University of Rome \\ “Sapienza”, Via di Grottarossa 1035, 00189 Rome, Italy. Email: claudio.andreetti@uniroma1.it.
}

\begin{abstract}
Superior outcomes after video-assisted thoracoscopic surgery (VATS) pulmonary lobectomy have been demonstrated, compared with thoracotomy, for patients affected by early-stage non-small cell lung cancer (NSCLC). However, in an era of rising health care costs and controlled resources, the overall medical cost of surgical procedures is measured by the hospitals' marketing offices. Several factors such as surgical technique, conversion rate, length of stay, post-operative complications occurrence and mainly the introduction of new surgical technologies (disposables and devices) could influence the cost-effectiveness of VATS procedures. However, increased operating room costs are counteracted by shorter hospital stay and reduced ward bed stays providing further resources for other patients. Thus, choosing a surgical approach and assessing whether a specific technique is cost-effective is mandatory for a modern thoracic surgeon to justify its expenses. This review tries to reach a conclusion by comparing all recent studies reporting a cost analysis for VATS lobectomy, especially for new technologies used for VATS lobectomy. Nevertheless, no sufficient evidences are published to assess VATS new technologies sustainability and further cost analyses are necessary before VATS lobectomy expense is deemed justified.
\end{abstract}

Keywords: Video-assisted thoracoscopic surgery (VATS) lobectomy; cost-effectiveness; technologies

Received: 06 July 2017; Accepted: 04 September 2017; Published: 28 October 2017.

doi: 10.21037 /jovs.2017.09.05

View this article at: http://dx.doi.org/10.21037/jovs.2017.09.05

A terrific interest has been generated by the video-assisted thoracic surgery (VATS) approach to lung lobectomy in recent years. Less morbidity and faster recovery after VATS lobectomy (VATSL) have been proved compared to conventional thoracotomy approach. However, the VATS new technologies costs have been discussed about whether this minimally invasive surgical approach for lung surgery may compromise hospital sustainability. The main issue is often expressed concerning the cost of stapling devices and increased operative time. The importance of debating this issue has been addressed not only to determining how patients are operated on, but to understanding how new technologies impact on hospital costs.
The value of health care, a concept equivalent to the outcomes achieved relative to money spent is gaining a terrific relevance in the current health care concept all over the world. As a result, health care reimbursement schemes will be undoubtedly changing over the next few years, and the current fee-for-service reimbursement scenario is being replaced by a bundled payment strategy for procedural. The value of health care increases when risk-adjusted clinicalfinancial data are understood to better design financial arrangements. Awareness of such factors makes surgeons implemented for quality improvement attitude and focused resource use.

This review helps to obtain the prospect of the 
experience of how VATS cost analysis evolved over the years. Systematic clinical evidence production after the increase of technological sophistication is required to precisely evaluate the VATS costs and to identify potential risk factors raising costs. Huge gaps in the evidence about VATS costs still remain reviewing the current literature, especially evidence regarding new technologies costs analysis. Moreover, a reduction of inpatient costs for VATS has not been uniformly demonstrated. Few authors have specifically considered the economic aspect of new technologies for VATSL and no detailed comparisons on new instruments and devices for VATS procedures in terms of costs are present. Hence, at the present time, there are not secure standardized data to judge that VATS should not be performed because of high costs. Instead, it is necessary surgeons to continue to generate good clinical evidence to assess it.

As following, costs/effectiveness results of main series comparing major lung resections through VATS $v s$. thoracotomy published in the last 17 years are reported (Table 1).

Rodgers-Fischl et al. (1) in 2017 reviewed 109 consecutive operations for all patients undergoing thoracotomy or VATSL performed at the University of Kentucky Chandler Medical Center.

The overall operative procedure time (170.6 vs. $196.3 \mathrm{~min}$ ), postoperative length of stay (LOS) $(5.7 \mathrm{vs}$. 5.5 days), number of lymph nodes sampled (6.2 vs. 7.0), and time spent in the intensive care unit (2.1 vs. 2.4 days) did not vary between both groups. The average cost per procedure did not vary significantly $-\$ 14,003.61$ compared with $\$ 15,588.11$ for thoracotomy and VATS, respectively. They concluded that VATS group was associated with no reduction in postoperative LOS and a nonsignificant reduction in the amount of time spent in the intensive care unit. Postoperative perception of pain did not vary between either group. Pain perception did, however, correlate strongly with time from operation. Cost did not vary significantly between both groups, with VATS being equivalent to thoracotomy in terms of cost.

Droghetti et al. (2) in 2017 evaluated the direct costs of pulmonary lobectomy hospitalization, comparing two different surgical devices for the interlobar fissures division: stapler (ST) vs. electrocautery and hemostatic sealant patch (ES). Use and maintenance of technology, equipment and operating room; administrative plus general costs; and 30-day use of post-surgery hospital resources were considered for 40 patients. Hospital perspective was used to conduct the cost analysis. A patient undergoing pulmonary lobectomy costs $€ 9,744.29 / \$ 11,559.9$ on average. This data could vary from $€ 9,027 / \$ 10,709.1$ (using ES) to $€ 10,460 / \$ 10,709.1$ (using ST). In the ES group, the mean time of hospital stay (11.0 vs. 14.3 days) and costs were significantly affected by the overall lower incidence $(50 \%$ vs. $95 \%, \mathrm{P}=0.0001)$ and duration of air leakage (1.7 vs. 4.5 days, $\mathrm{P}=0.0001$ ). The lower incidence of complications in the ES group also determined a substantial cost saving. The main key cost burden was staff employment (42\%), then consumables (34\%) and operating room costs $(12 \%)$. There was an overall saving of around $€ 1,432.90 / \$ 1,699.7$ when using ES patch for each pulmonary lobectomy. The authors concluded that air leakage incidence and duration, as well as hospitalization rates could significantly be reduced by the ES use.

Medbery et al. (3) in 2014 showed the VATSL cost variability by analyzing 149 VATSLs for lung cancer. Median LOS was 4 days, with 30-day mortality and morbidity rates of $0.7 \%$ and $37.6 \%$, respectively. Mean operative and postoperative costs per case were $\$ 8,492.31$ $( \pm \$ 2,238.76)$ and $\$ 10,145.50( \pm \$ 7,004.71)$, respectively, resulting in an average overall hospital cost of $\$ 18,637.81$ $( \pm \$ 8,244.12)$ per patient. Patients with chronic obstructive pulmonary disease and coronary artery disease, as well as post-operative urinary tract infections and blood transfusions, were associated with statistically significant variability in cost.

They concluded that variability in cost associated with VATSL was driven by assorted patient and clinical variables.

Farjah et al. (4) in 2014 analyzed the complications after VATS pulmonary resection leading to increased costs of care. The authors analyzed 90-day costs comparing VATSL $v s$. open lobectomy (OL) and investigating if differential health care use after discharge might cause any observed differences in costs. A cohort study (20072011) of patients with lung cancer who had undergone resection was performed using Market Scan, a nationally representative sample of persons with employer-provided health insurance. Total costs reflected payments made for inpatient, outpatient, and pharmacy claims up to 90 days after discharge. Out of 9,962 patients, 31\% underwent VATSL. Compared with thoracotomy, VATS offered a lower rate of prolonged LOS (PLOS) (3.0\% vs. 7.2\%; $\mathrm{P}<0.001)$, 90-day emergency department (ED) admission $(22 \%$ vs. $24 \% ; \mathrm{P}=0.005)$, and 90 -day readmission $(10 \%$ vs. $12 \%$; $\mathrm{P}=0.026)$. VATSL showed a lower risk-adjusted 90 -day costs of $\$ 3,476$ ( $\mathrm{P}=0.001)$. Differential rates of PLOS appeared to explain this cost difference. After adjustment for PLOS, 


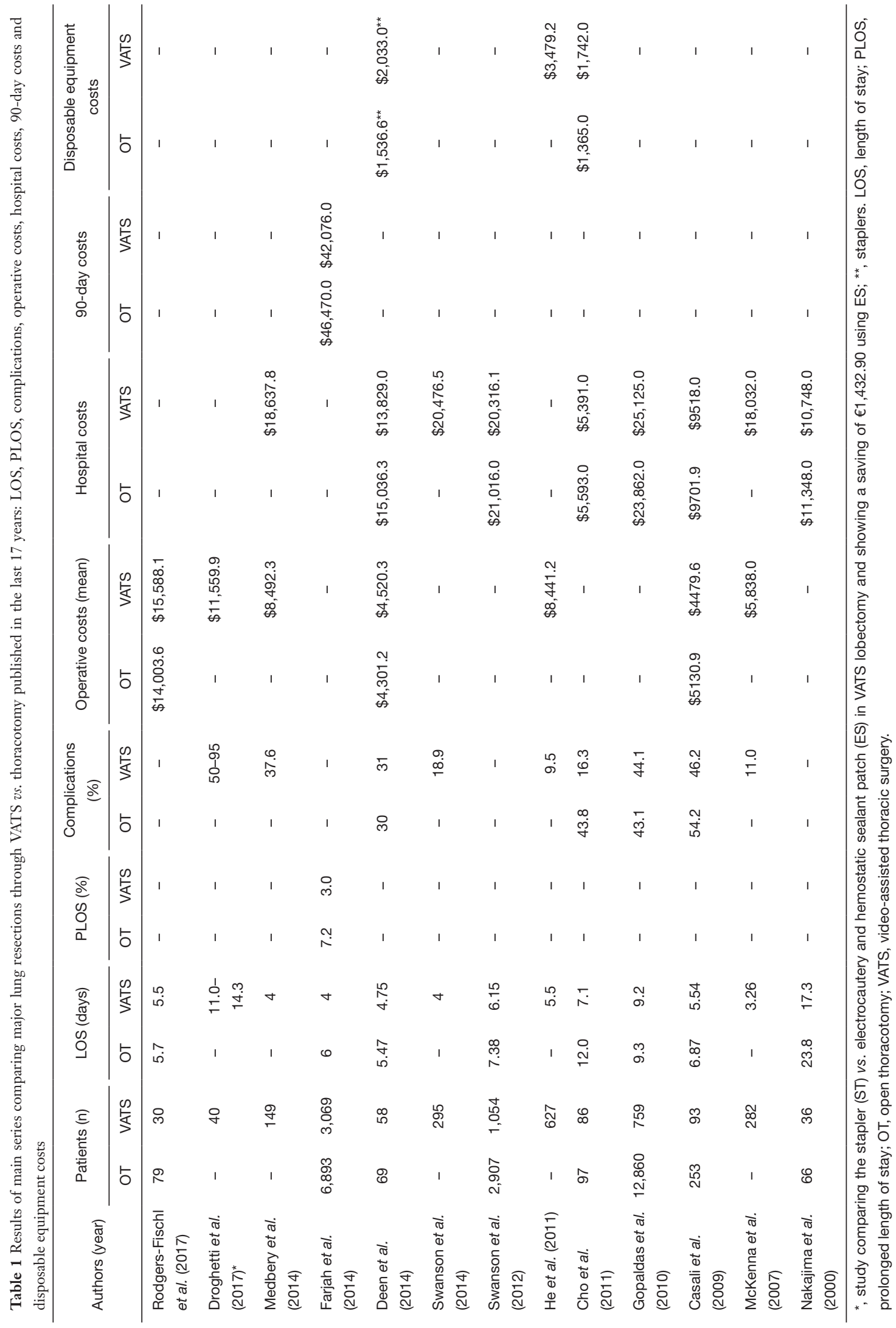


costs were $\$ 1,276$ lower for VATS, but this difference was not significant $(\mathrm{P}=0.125)$. In the fully adjusted model, PLOS was associated with the highest cost differential $(+\$ 50,820$; $\mathrm{P}<0.001)$. VATSL showed a lower 90 -day costs, apparently consecutive to lower rates of PLOS.

Deen et al. (5) in 2014 retrospectively defined the cost of care for lobectomy and segmentectomy, comparing thoracotomy $(\mathrm{n}=69)$, VATS $(\mathrm{n}=58)$ and robotic approaches $(\mathrm{n}=57)$, in patients affected by early stage lung cancer, carcinoid, or metastatic foci who had anatomic resection. Ten categories were considered to calculate direct hospital cost. The authors distanced the robotic and VATS cases for capital depreciation. Key costs were varied in a sensitivity analysis. No statistically significant difference in overall cost between VATS and open cases $(\Delta=\$ 1,207)$ or open and robotic cases $(\Delta=\$ 1,975)$ were shown. Roboticspecific supplies made robotic cases cost $\$ 3,182$ more than VATS $(\mathrm{P}<0.001)$. Intensive care unit, respiratory therapy and laboratories raised costs in thoracotomy cases. Thus, VATS was the least expensive surgical approach. Lessening robotic operative time, avoiding unnecessary laboratory examination and minimizing intensive care unit stays will help decrease direct hospital costs.

Swanson et al. (6) in 2014 performed a propensity score matched analysis comparing robot-assisted thoracic surgical (RATS) lobectomy with conventional VATSL and wedge resection. Using the Premier hospital database, patients undergoing lobectomy, segmental resection, or wedge resection through VATS were considered. Out of 15,502 patient reports analyzed, $96 \%(n=14,837)$ were performed without robotic assistance. Higher average hospital costs per patient was associated with the use of robotic assistance. The average cost of inpatient procedures with RATS was $\$ 25,040.70$ vs. $\$ 20,476.60$ for VATS $(\mathrm{P}=0.0001)$ for lobectomies and $\$ 19,592.40$ vs. $\$ 16,600.10(\mathrm{P}=0.0001)$ for wedge resections, respectively. Inpatient operating time were longer for RATS lobectomy than VATSL (4.49 vs. $4.23 \mathrm{~h} ; \mathrm{P}=0.0959)$ and wedge resection $(3.26$ vs. $2.86 \mathrm{~h}$; $\mathrm{P}=0.0003)$. No differences in LOS and adverse events were registered.

Swanson et al. (7) in 2012 compared VATS and OL procedures in terms of hospital costs and perioperative outcomes using The Premier Perspective Database (Premier Inc., Charlotte, NC, USA). A total of 3,961 patients undergoing pulmonary lobectomy through open $(n=2,907)$ or VATS $(n=1,054)$ approach. The open approach showed higher hospital costs than VATS; \$21,016 vs. \$20,316 $(\mathrm{P}=0.027)$. A significant association between surgeon experience and cost was proved adjusting for surgeon experience with VATS over the 6 months prior to each operation. Average costs ranged from $\$ 22,050$ for low volume surgeons to $\$ 18,133$ for high volume surgeons. Not significant cost differences by surgeon experience for open lobectomies were shown and both levels were measured at $\$ 21,000$. LOS for open was 7.83 vs. 6.15 days for VATS $(\mathrm{P}=0.000)$. Open procedures were shorter than VATS ( 3.75 vs. $4.09 \mathrm{~h}, \mathrm{P}=0.000$ ). The risk of adverse events was significantly lower in the VATS group [odds ratio (OR), 1.22; $\mathrm{P}=0.019]$. Therefore, the positive economic impact of VATSL was magnified as the surgeon's experience increased.

He et al. (8) in 2011 compared the outcomes and costs between complete VATS (c-VATS) or assisted VATS (a-VATS) major pulmonary resection in 1,058 patients affected by non-small cell lung cancer (NSCLC). Mean operative time was shorter in the a-VATS cohort $v s$. the c-VATS group $(\mathrm{P}=0.038)$. Overall survival $(\mathrm{OS})$ at 5 years was $55.3 \%$ [ $95 \%$ confidence interval (CI), $50.6-60.0 \%$ ] in the c-VATS group and $47.7 \%$ (95\% CI, $41.2-54.2 \%$ ) in the a-VATS group $(\mathrm{P}=0.404)$. Predictive factors for OS were gender, final pathology, TNM stage and $\mathrm{pT}$ status, according to multivariate analysis. The total cost of a-VATSL was lower than that of c-VATSL, but no differences were shown in postoperative results. a-VATS could be a particularly attractive option in developing countries due to its less expensive costs.

Cho et al. (9) investigated on the costs of VATSL $v$ s. OL, analyzing the impact of surgeon's experience level on VATSL cost.

Eight-six patients in a VATSL group and 97 patients in an OL group underwent surgery for lung cancer. Cost comparisons were performed for the VATSL $(n=86)$ and OL ( $\mathrm{n}=97)$ groups facing patients who had no complications and patients who showed complications considering the tumor location and the learning period of the surgeon. Postoperative complications occurred in $30.6 \%$ of patients (14 VATSL vs. 42 OL patients; $\mathrm{P}=0.05$ ). Chest tube duration (5.4 vs. 9.1 days; $\mathrm{P}=0.000$ ) and length of hospital stay (7.1 vs. 12.0 days; $\mathrm{P}=0.000)$ were shorter in VATSL group. No differences for the mean operative time was shown between VATSL and OL (145.8 vs. $136.4 \mathrm{~min}$; $\mathrm{P}=0.782)$. The total hospital cost was lower for VATSL than for OL both among all patients $(\$ 5,391$ vs. \$5,593, respectively) and non-complicated patients $(\$ 4,684$ vs. $\$ 4,769$, respectively). Moreover, the right lower lobe, left upper lobe and left lower lobe were less expensive than all 
the other lobes in terms of hospital cost when lobectomy was performed through VATS. No significantly differences in costs were shown between the two groups according to surgeons' learning periods, except for the cost of anesthesia.

Gopaldas et al. (10) in 2010 used a national database to compare open $v s$. VATSL results. They considered 13,619 discharge records of patients who underwent pulmonary lobectomy through thoracotomy $(\mathrm{n}=12,860)$ or VATS $(\mathrm{n}=759)$. Mortality rates $(3.1 \%$ vs. $3.4 \% ; \mathrm{P}=0.67)$, LOS (9.3 \pm 0.1 vs. $9.2 \pm 0.4$ days; $\mathrm{P}=0.84$ ), hospitalization costs $(\$ 23,862 \pm 206$ vs. $\$ 25,125 \pm 1,093 ; \mathrm{P}=0.16)$, wound infection rates $(0.8 \%$ vs. $1.3 \% ; \mathrm{P}=0.15)$, pulmonary complications (32.2\% vs. $31.2 \% ; \mathrm{P}=0.55)$ and cardiovascular complications ( $3.4 \%$ vs. $3.9 \% ; \mathrm{P}=0.43$ ) were similar between the two groups, with no significant differences. Nevertheless, the VATS group was significantly impacted by the higher incidence of intraoperative complications than the thoracotomy group at multivariate analysis (OR, 1.6; $95 \%$ CI, 1.0-2.4; $\mathrm{P}=0.04)$. Moreover, a higher number of patients with annual income greater than $\$ 59,000$ underwent VATSL than patients with income less than $\$ 59,000$ (35.7\% vs. $25.4 \% ; \mathrm{P}<0.0001)$. Thus, intraoperative complications were more frequent (1.6 times) in patients who underwent VATSL than patients who underwent OL. However, short-term mortality, LOS, and hospitalization costs were similar between the two groups of patients. There seemed to be a socioeconomic disparity between VATS and open thoracotomy patients.

Casali et al. (11) in 2009 investigated the overall economic sustainability of a VATS program comparing the costs of VATS and OL. Direct medical costs [disposables, theatre time, high dependency (HDU) unit stay, hospital stay] were assessed and stratified by lobectomy type. Mean theatre cost for a VATSL was $\$ 2,533 \pm 230$ vs. $\$ 1,280 \pm 54$ for a thoracotomy lobectomy $(\mathrm{P}=0.00001)$. Mean HDU cost was respectively $\$ 1,713 \pm 236$ and $\$ 2,571 \pm 80$ for a VATS and a thoracotomy lobectomy $(\mathrm{P}=0.00001)$. Mean cost of hospital stay for a VATSL was $\$ 3,776 \pm 281$ vs. $\$ 4,325 \pm 154$ for an open one $(\mathrm{P}=0.00001)$. The overall cost was less for a VATSL $(\$ 8,023 \pm 565)$ than the cost for an OL $(\$ 8,178 \pm 167$, $\mathrm{P}=0.0002)$. VATS bilobectomy was slightly more expensive than an open one: $\$ 8,702 \pm 350$ vs. $\$ 8,655 \pm 466$ with no significant difference $(\$ 47, \mathrm{P}=\mathrm{ns})$. Shorter hospital stay neutralized increased theatre costs (disposables and time). Importantly, the reduced HDU and ward bed stays allow free resources for new cases.

McKenna et al. (12) in 2007 aimed to show if a fasttracking protocol after VATSL provided a minimal LOS without raising morbidity and mortality or causing a readmission to the hospital. VATSLs were performed without routine postoperative laboratory examination or chest X-Rays. The chest tubes were removed when the quantity of fluid drained was less than $300 \mathrm{~mL}$ in a $24 \mathrm{~h}$ period and there was no air leak present. The patient was discharged home with a Heimlich valve if air leak was present. All procedures were performed by a single surgeon. Mean LOS was 3.26 days. Seven of 282 patients (2.5\%) were discharged with a Heimlich valve. Only one patient died. No complications were registered in 251 patients (89\%). Two patients were readmitted to the hospital. No chest tubes were reinserted. The hospital LOS impacted the hospital's gross margin for a patient with Medicare coverage. The main impact for the gross margin per room in a year was the reduction of the hospital LOS from 7 to 2 days. The Medicare diagnosis-related group (DRG) 075 paid approximately $\$ 24,000$ for a lobectomy, independently the LOS was 2 or 7 days. The hospital cost for the stay increased slightly with each additional day in the hospital. Thus, the gross margin was slightly reduced for each less day in the hospital. Over the course of an entire year, a large difference in gross margin was registered. A LOS of 2 days was translated in 3.5 lobectomy patients who could use that hospital room instead of one patient if the LOS was 7 days. A huge difference in the gross margin for that hospital room and a substantial savings in the financial bottom line for hospitals was sustained by the shorter LOS.

Nakajima et al. (13) in 2000 analyzed the VATSL costs in Japan, where the health care reimbursement is completely different from European or American countries. In this series, videothoracoscopic lobectomy or partial resection of the lung instead of an open thoracotomy was preferred for patients with more comorbidities or a not good performance status affected by lung carcinoma. Out of 102 patients considered for the analysis, 79 patients had primary lung carcinoma, and 23 had metastatic lung carcinoma. Sixtysix open thoracotomies and 36 thoracoscopic surgeries were performed. The mean hospital cost for the total of patients was $\$ 11,348$. The total charges accrued in the operating room amounted to $63 \%$ of the hospital costs. LOS was significantly shorter in the VATS group (17.3 days) compared with the thoracotomy group (23.8 days). Laboratory examinations, anesthesia, disposable equipment, and hospitalization costs were significantly higher in patients who underwent open thoracotomy compared with the patients who underwent videothoracoscopy. No statistically significant differences in the costs for medication or surgical fees between the 
two groups were shown. They were lower than the charges for patients undergoing open thoracotomy at the authors' hospital. The lower hospital charges for patients undergoing videothoracoscopic surgery were attributable mainly to the less invasive nature of VATS procedures, lessening the risk of postoperative complications in relatively poor health patients.

In conclusion, to date, when a VATS approach is used, significant cost savings seem to be reported compared to a thoracotomy for lung cancer surgery $(14,15)$. If concern about the cost of VATS equipment is raised, the VATS approach is clearly favored over a thoracotomy $(16,17,18)$ offering a significant hospital savings associated with better outcomes, particularly when an experienced surgeon performs the surgery. Moreover, in the era of cost containment, a fast-tracking protocol is suggested to lessen cost and shorten the LOS after a lobectomy. However, no standardized items were followed to perform a cost analysis, thus all reports present in literature are extremely different, evaluating not similar and not comparable aspects of VATSL expense. Furthermore, no specific numbers are reported for new instruments or technological devices applied for VATSL, making really challenging the cost analysis in the new era of minimally invasive surgery. Prospective studies considering standardized variables and economic measurement tools are necessary to better understand the real burden of new technologies for VATSL.

\section{Acknowledgements}

The authors acknowledge Dr. M. Silvi, Data Manager, Division of Thoracic Surgery, Sant'Andrea Hospital, Faculty of Medicine and Psychology, University of Rome "Sapienza".

\section{Footnote}

Conflicts of Interest: The authors have no conflicts of interest to declare.

\section{References}

1. Rodgers-Fischl PM, Martin JT, Saha SP. Video-Assisted Thoracoscopic versus Open Lobectomy: Costs and Outcomes. South Med J 2017;110:229-33.

2. Droghetti A, Marulli G, Vannucci J, et al. Cost analysis of pulmonary lobectomy procedure: comparison of stapler versus precision dissection and sealant. Clinicoecon
Outcomes Res 2017;9:201-6.

3. Medbery RL, Perez SD, Force SD, et al. Video-assisted thoracic surgery lobectomy cost variability: implications for a bundled payment era. Ann Thorac Surg 2014;97:168692; discussion 1692-3.

4. Farjah F, Backhus LM, Varghese TK, et al. Ninety-day costs of video-assisted thoracic surgery versus open lobectomy for lung cancer. Ann Thorac Surg 2014;98:191-6.

5. Deen SA, Wilson JL, Wilshire CL, et al. Defining the cost of care for lobectomy and segmentectomy: a comparison of open, video-assisted thoracoscopic, and robotic approaches. Ann Thorac Surg 2014;97:1000-7.

6. Swanson SJ, Miller DL, McKenna RJ Jr, et al. Comparing robot-assisted thoracic surgical lobectomy with conventional video-assisted thoracic surgical lobectomy and wedge resection: results from a multihospital database (Premier). J Thorac Cardiovasc Surg 2014;147:929-37.

7. Swanson SJ, Meyers BF, Gunnarsson CL, et al. Videoassisted thoracoscopic lobectomy is less costly and morbid than open lobectomy: a retrospective multiinstitutional database analysis. Ann Thorac Surg 2012;93:1027-32.

8. He J, Shao W, Cao C, et al. Long-term outcome and costeffectiveness of complete versus assisted video-assisted thoracic surgery for non-small cell lung cancer. J Surg Oncol 2011;104:162-8.

9. Cho S, Do YW, Lee EB. Comparison of costs for videoassisted thoracic surgery lobectomy and open lobectomy for non-small cell lung cancer. Surg Endosc 2011;25:1054-61.

10. Gopaldas RR, Bakaeen FG, Dao TK, et al. Video-assisted thoracoscopic versus open thoracotomy lobectomy in a cohort of 13,619 patients. Ann Thorac Surg 2010;89:1563-70.

11. Casali G, Walker WS. Video-assisted thoracic surgery lobectomy: can we afford it? Eur J Cardiothorac Surg 2009;35:423-8.

12. McKenna RJ Jr, Mahtabifard A, Pickens A, et al. Fasttracking after video-assisted thoracoscopic surgery lobectomy, segmentectomy, and pneumonectomy. Ann Thorac Surg 2007;84:1663-7; discussion 1667-8.

13. Nakajima J, Takamoto S, Kohno T, et al. Costs of videothoracoscopic surgery versus open resection for patients with of lung carcinoma. Cancer 2000;89:2497-501.

14. Lacin T, Swanson S. Current costs of video-assisted thoracic surgery (VATS) lobectomy. J Thorac Dis 2013;5 Suppl 3:S190-3.

15. Spartalis E, Mantonakis E, Athanasiou A, et al. Lobectomy by Video-Assisted Thoracic Surgery or MuscleSparing Thoracotomy for Stage 1 Lung Cancer: Could Cost-Effectiveness Give the Answer? J Am Coll Surg 
2015;221:890.

16. Lewis RJ, Caccavale RJ, Sisler GE, et al. Is video-assisted thoracic surgery cost effective? N J Med 1996;93:35-41.

17. Walker WS, Casali G. The VATS lobectomist: analysis of costs and alterations in the traditional surgical working

doi: 10.21037/jovs.2017.09.05

Cite this article as: Menna C, Ibrahim M, Rendina EA, Venuta F, Andreetti C. Cost/efficacy evaluation of the technologies applied to video-assisted thoracoscopic surgery lobectomy. J Vis Surg 2017;3:152. pattern in the modern surgical unit. Thorac Surg Clin 2008;18:281-7.

18. Hunt I, Chuck A, Tsuyuki R, et al. Influence of conversion on cost of video-assisted thoracoscopic lobectomy. Eur J Cardiothorac Surg 2010;37:249-50; author reply 250-1. 